\section{Die Geschlechtsreife bei den farbigen Menschenrassen.}

Von H. Fehlinger, München.

Es ist bis heute noch die vorherrschende Meinung, dab das Klima von exheblichem EinfluB auf den Eintritt der Geschlechtsreife sei. So sagt Rudolf Martin in seinem kürzlich erschienenen Lehrbuch der Anthropologie $\mathrm{e}^{\mathrm{x}}$ : ,In den Tropen lebende Rassen wachsen rascher und sind früher körperreif als Rassen der gemäßigten Zone. Die Ursache dafür liegt ohne Zweifel in dem früheren respektive späteren Eintritt der Pubertatsentwicklung."

Soweit Japaner in Betracht kommen, hat E. Baelz schon 1901 ihre angebliche Frubreife bestritten; er fand zwar, daß das Wachstum beider Geschlechter in Japan früher absehlieBt als in Europa, aber die Entwicklung des weiblichen Geschlechts geht dort trotzdem nicht rascher vor sich. Nach ubereinstimmenden Angaben von Lehrerinnen verschiedener Mädchenschulen werden die japanischen Müdchen spüter geschlechtsreif als die europäischen, und die Mischlingsmädchen nehmen eine Mittelstellung ein ${ }^{2}$.

Seitdem sind verläßliche Angaben über den Eintritt der Geschlechtsreife bei den farbigen Rassen recht selten gemacht worden.

Sehr wichtiges Material hat $O$. Reche auf Matupi (Neu-Pommern, Melanesien) mit Hilfe der dortigen katholisehen Mission gesammelt. Reohe fand ${ }^{3}$, dab der Waohstumsrhythmus der Melanesier ungefähr dem der Europüer entspricht, nur ist das ganze Wachstum um einige Jahre frïher beendet: „Mit Beginn des 17. Lebensjahrs scheint in der Hauptsache bei den Mädehen, mit dem 18. bei den Knaben das Größenwachstum abgeschlossen zu sein." Aber bezüglich des Eintritts der Pubertät führten Reches Untersuchungen zu dem iuberraschenden Ergebnis, daß alle Matupimädchen, mit Ausnahme eines siebzehnjührigen, noch nicht menstruiert hatten. Reche bemerkt, dieses auffällig späte Eintreten der Menstruation sei übrigens auch den Missionaren bekannt, weil diese zur Verhinderung der allzu fruthzeitigen Eheschließung nur nach bereits erfolgtem Eintreten der Menstruation der Braut die Ehebewilligung erteilen. Reches Ergebnis widerspricht auf das krasseste all dem, was man bisher annahm: "Die Pubertät tritt bei diesen Tropenbewohnern nicht nur nioht frihher, sondern sogar später ein, als bei dem in gernäBigtem Klima lebenden Europäer. Sehr wichtig ist nun, daB bei den Matupi-Eingeborenen die Pubertät mit der markantesten Stelle der Wachstumskurve zusammenfällt, nämlich mit der Beendigung des GröBenwachstums. Die Pubertät setzt in dem Moment ein, wo das GröBenwachstum aufhört. Es sieht fast so aus, als ob der Eintritt der Geschlechtsreife alle Kraft absorbiere und ein weiteres Wachstum verhindere. Europäer verhalten sich bekanntlich in dieser Beziehung ganz anders; bei ihnen fält der

1) Martin, Lehrbuch der Anthropologie, S. 232 und 234. Jena, 1914. Gustav Fischer.

2) Baelz, Die körperlichen Eigenschaften der Japaner, Bd. 2, S. 37; ders., Das Wachstum der Geschlechter zur Pubertätszeit. Verhandl. d. Berl. Ges. f. Anthropologie, 1901, S. 211.

3) Reche, Untersuchungen uber Wachstum und Geschlechtsreife bei melanesischen Kindern, Korrespondenzblatt d. d. Ges. f. Anthropologie usw., 41. Jahrg., Nr. 7.
Eintritt der Pubertat in die Periode der zweiten Streckung ${ }^{1}$, also weit vor Beendigung des Größenwachstums." (Reche.)

Es scheint, daß das bei den Europäern bestehende Verhältnis den ursprünglichen Zustand anzeigt, da auch bei der MehrzahI der Sängetiere die Pubertät vor dem AbschluB des GröBenwachstums eintritt.

Reche berichtet ferner, daB bei den Matupikindern, dem späten Eintreten der Pubertat entsprechend, die sekundären Geschlechtsmerkmale auffallend spät zur. Ausbildung kommen, was der Hauptgrund dafir ist, „daß die Knaben und Mädchen, zumal bei ihrer Klein. heit, selbst in ihren späteren. Kinderjahren, auffallend jung aussehen, dab man si stets jünger einschätzt, als sie in Wirklichkeit sind. . . Erst bei den $16 \mathrm{jährigen}$ Matupimädchen zeigte sich der erste Ansatz des Utberganges von der Areolomamma zur Knospenbrust; die Entwicklung der Brust scheint also mit der ersten Menstruation ungefähr zusammenzufallen." Axillar-

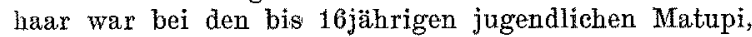
mit einer Ausuahme, noch gar nicht vorhanden und bei den 17jährigen recht spärlich, obzwar es bei älteren Erwachsenen meist recht reichlich ist. Auch von Bartwuchs, der bei älteren Männern ziemlich stark ent wickelt ist, war bei den bis 17 jährigen Jünglingen keine Spur. - Dazu möchte ich bemerken, dab die späte Differenzierung der sekundären Geschlechtsmerkmale auch bei anderen farbigen Rassen auffällt, wie z. B. bei den Philippinern und den Indonesiern.

In Dewtsoh-Neuguinea tritt die Geschlechtsreife ebenfalls spät ein. Richard Neuhau ${ }^{2}$ ) sagt, nach Angabe von Missionaren, die schon lange unter den Papua leben, stellt sich auf Tami und bei den Jabim die ersto Periode gewöhnlich mit dem 15. oder 16. Lebensjahr ein. Junge männliche Personen sehen bis zum 16. Jahre sehr unentwickelt aus. Neuhaup bäl die Spätreife für eine Folge schlechter Ernährung, obzwar aus seiner sonstigen Darstellung nicht hervorgeht, dab die Lebensverhältnisse der Papua besonders ungünstig seien.

A. E. Jenks berichtet von Nord-Luzon ${ }^{3}$, daß sowohl Knaben wie Mädchen beim Stamm der Igoroten die Pubertät in einem späten Alter erreichen, u. zw. ge wöhnlich $z$ wischen 14 und 16 Jahren. Die unter den Igoroten angesiedelten zivilisierten Nokanoleute behaupteten bestimmt, daß die Mädehen nicht menstruierten; bevor sie nicht das 16. oder 17. Lebensjahr er. reicht haben. Ein beträchtlicher Irrtum hinsichtlich des Alters scheint ber diesen Leuten, die schon lange unter europäischem Einfluß stehen, ausgeschlossen zu sein.

Eugen Fischer macht uber die Bastards in Deutsohsüdwestafrika folgende Angaben ${ }^{4}$ ): „In einer Familie menstruierten von den sechs Töchtern fünt zum ersten Male mit 15 Jahren, eine mit 16 Jahren. Eine Bastardfrau hatte seinerzeit mit 17 Jahren zuerst menstruiert, von ihren Töchtern drei mit je 13 Jahren, die vierte, die kränklich (chlorotisch?) war, mit 17 Jahren. Eine andere Bastardfrau, die selber mit 15 Jahren die erste Menstruation hatte, hat von einem weiBen Manne zwei Töchter, die mit 16 und 17 Jahren reif wurden. Ein Mädchen - mit deutlicher Chlorose - gab an, mit 16 Jahren die erste Menstruation gehabt zu haben, ihre

1) Bei Knaben das 12. bis einsehlieslich 16., bei Mädchen das 11. bis einschlieBlich 14. Jahr.

2) Neuhan $\beta$, Deutseh-Neuguinea, Bd. 1. Berlin 1911. 1905 ,

3) Jenks, The Bontoc Igorot, S. 46 u. 66 . Manila

4) Fischer, Die Rehoboter Bastards und das Bastardierungsproblem beim Merischen, S. 123 . Jena 1913. 
Schwester sogar erst mit 18 Jahren." - Von drei Mädchen weil Fischer, dab sie mit 16, 14 und 13 Jahren reiften. L. Schultze (von Fisoher zitiert) berichtet, daß bei den Hottentotten die erste Menstruation in der Regel zwischen dem 13. und 15. Lebensjahre eintritt; auf welchen Grundlagen diese Angabe beruht, wird nicht gesagt.

Uber die Neger liegen leider gar keine auf diesen Gegenstand bezügliehen Angaben vor. Die bei den Negern vielfach üblichen Reifezeremonien geben keinen Anhalt über das tatsächliche Alter beim Eintritt der Reife.

Das Reifealter von Indianermädohen im Sudwesten der Vereinigten Staaten suchte Ale š Hrdlioka nach der Körperlänge der Mädchen zu bestimmen, weil tatsächliche Altersangaben mangeln ${ }^{1}$ ). Diese Methode ist nicht einwandfrei, denn es steht fest, daß die schon geschlechtsreifen Individuen beträchtlich größer sind als gleichaltrige noch nicht geschlechtsreife Individuen ${ }^{2}$ ). Hrdlicka fand, dab von den Untersuchten, die im 12.-13. Lebensjahr standen, bei den Apachenmädchen ein Drittel und bei den Pimamädchen sogar drei Viertel bereits menstruiert hatten. In der Altersklasse 13-14 Jahre hatten vier Fünftel der Apachenund neun Zehntel der Pimamädchen schon menstruiert und von 46 alteren Mädchen war nur eins noch nicht geschlechtsreif. Die ersten Zeichen der Entwicklung der Mammae merkte Hrdlička bei angekleideten Indianermädchen, deren Alter er auf 11-12 Jahre schätzt. Aber erst mit $15-17$ Jahren nimmt der Mädchenkörper die typisch weiblichen Formen an; bis dahin hat er, wie Hrdličlca meint, ,eine etwas männliche Form". Bei den Jünglingen fängt der Bart mit etwa dem 15.-16. Jahr zu wachsen an. (Tch habe den Eindruck, daß Bralička das Alter der Kinder und jungen Leute unterschätzt.) Das Klima ist im Wohngebiet der Apache- und Pimaindianer gemäBigt; die Tage sind in den tiefer gelegenen Regionen entschieden heis, aber die Nächte sind sogar in diesen Regionen und auch im Sommer gewöhnlich kalt.

Zum Vergleich soll bemerkt werden, daß nach $H . P$. Bowditchs Untersuchungen ${ }^{3}$ ) in Boston nahezu vier Fünftel der weißen in Amerika geborenen Mädchen zwischen dem 13. und 17. Jahr reif werden. Verbältnismäßig am häufigsten tritt die Reife zwischen dem 14. und 15. Jahr ein, aber über $40 \%$ von insgesamt 575 Mädchen hatten mit vollendetem 15. Jahr noch nicht menstruiert.

Uber den Eintritt der Pubertatt bei Europäerinnen liegen viele, aber zum Teil einander widersprechende Angaben vor. Auf Grund eines 10500 Frauen umfassenden Materials stellte $R$. Schäffer ${ }^{*}$ für Deutschland das mittlere Alter des Menstruationsbeginnes mit ca. 153/4 Jahren fest; in $53,3 \%$ der Falle traf der Menstruationsbeginn auf das 14. bis 16. Lebensjahr und in $85,1 \%$ der Fäle auf das 13 . bis 19. Lebensjabr. Reche meint, daß auf Grund von Literaturangaben der Men* struationsbeginn der Europäerinnen am häufigsten in das 14. Lebensjahr fällt. Bei sieben siddeutschen Mädchen, von welchen ich sichere Angaben babe, begann die Menstruation ausnahmslos im 14. Lebensjahr; darwnter befinden sich je zwei und drei Sehwestern. An

\footnotetext{
1) Irdlička, Physiological and Medical Observations among the Indians, S. 125-129. Washington 1908.

2) Martins 'Tehrbuch, S. 232.

3) Bowditoh, The Growth of Children (8th Ann. Rept. State Board of Health, S. 12, Boston 1877).

4) Schäfer, Uber das Alter des Menstruationsbeginns. Arch. f. Gynäkologie Bd, 84 .
}

pathologische prämature Geschlechtsentwicklung wäre nur in einem dieser sieben Falle $z u$ denken, da in den ibrigen das auffallendste Zeichen einer solchen Mntwicklung (Kürze der Extremitäten bei Länge des Rumpfes) nicht vorhanden ist.

Innerhalb einer und derselben Rasse scheinen die Lebensbedingungen von großem Einflua auf das Reifealter wie auf die körperliche Entwicklung überhaupt zu sein. Ungiunstige Lebensverhältnisse bewirken eine Verlangsamung der Reife, günstige Lebensbedingungen dagegen beschleunigen sie ${ }^{\mathrm{T}}$ ). Hierauf dürfte vor allem die Tatsache beruhen, daß der Eintritt der Pubertät individuell um mehrere Jahre verschieden sein kann.

Eine sichere Erklärung der in der Rasse begründeten Verschiedenheiten des Pubertätsalters haben wir noch nicht. Reche sagt (a. a. O.), ,es wäre denkbar, daß die für eine tropische Rasse (wie die Melanesier) charakteristische späte Geschlechtsreife durch den auf viele Generationen einwirkenden ungünstigen Einflub eines zu heiBen Klimas oder von ständiger Unterernährung allmählich erworben wurde".

Dagegen führt der amerikanische Arzt $C . \quad E$. Woodruff ${ }^{2}$ ) das verschiedene Pubertätsalter der Nordund Sideuropäerinnen darauf zurïck, daß bei den in bezug anf geschlechtliche Moral strengeren Nordeuropäern die Ausmerzung der zu frühem Geschlechtsverkehr neigenden Personen seit langem mit großer Schärfe vor sich gehe, während sie in Südeuropa erhal ten blieben. Durch diese Auslese mußte selbstverständlich das Pubertätsalter hinaufgeräckt werden. - Ähnliches könnte auch von den farbigen Rassen angenommen. werden, bei welchen in einem noch nicht allzu weit zurückliegenden Abschnitt ihrer Geschichte der frühzeitige Geschlechtsverkehr verpönt war. Die ethnographische Literatur zeigt a.t sehr vielen Beispielen, daß gerade die sogen. primitiven Volker gegen solche Leute mit großer Strenge vorzugehen pflegen, die gegen die bestehenden Regeln des Geschlechtsverkehrs verstoßen. Von diesem Standpunkt aus betrachtet, hätten die Verschiedenheiten des Pubertätsalters als Folgen der Anpassung an gewisse gesellschaftliche Zustände zu gelten.

\section{Besprechungen.}

Kaye, G. W. C., $X$ Rays, an introduction to the study of Röntgen Rays. London, Longmans, Green \& Co., 1914. 250 S. Preis 5 sh.

Das Kayesche Buch ist im besten Sinne des Wortes eine Einfübrung in das Studium der Röntgenstrahlphysik, für den Praktiker und Mediziner sowohl wie für den experimentellen und theoretischen Physiker. In Anbetracht des sehr heterogenen Leserkreises, den das Buch erwarten darf, beschränkt sich der Verfasser nicht ängstlich auf das engere Thema, sondern sucht uberall durch Betrachtung verwandter Phänomene den Gesichtskreis des Lesers so zu weiten, daB er an Bekanntes anknüpfen kann. So ist $z$. B. dem Buch eine allgemeine Ubersicht über die Vorgänge in Entladungsrohren bei verschiedenem Druck des Gases vorausgeschickt, ferner wird kurz über die Bestimmung von $\mathrm{e} / \mathrm{m}$ an KathodenstrahIen und $J$. J. Thomsons Kanal-

1) Vgl. Mariins Lehrbuch der Anthropologie, S. $23 \overline{5}$, sowie Busohans Menschenkunde, S. 231:

2) Woodruff, Expansion of Races, S. 191 u. ff. New York 1909. 